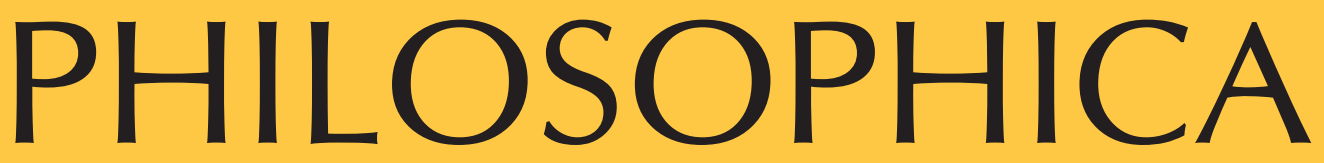

\author{
FILOSOFIA E MEDICINA \\ SOBRE DOR E SOFRIMENTO
}

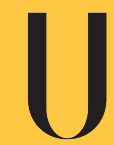

$$
\begin{gathered}
\text { LISBDA } \\
\text { UNIVERSIDADE } \\
\text { DE IISBOA }
\end{gathered}
$$

Departamento de Filosofia

Faculdade de Letras da Universidade de Lisboa Centro de Filosofia da Universidade de Lisboa 


\title{
La Respuesta al Problema del no-ser en La Metafísica de Aristóteles
}

\author{
Maria Carmen Segura Peraita ${ }^{1}$ \\ (Facultad de Filosofía, Departamento de Lógica \\ y Filosofía Teórica, Madrid)
}

El objetivo de este trabajo es proponer una lectura de la Metafísica de Aristóteles entendida como respuesta al inquietante y esquivo problema del no-ser, que había tenido en vilo y atrapado en aporías al pensamiento anterior. Esta orientación revela la vigencia de los planteamientos ontológicos aristotélicos para el presente, porque también hoy el movimiento, la diferencia, la inidentidad y el tiempo constituyen problemas filosóficos fundamentales.

Es sabido que Aristóteles elabora su ontología en diálogo y confrontación con Platón (Sofista y Parménides), aunque también con los sofistas, los físicos y los megáricos. En esta ponencia se apuntará a determinados aspectos del debate mencionado en la medida que contribuyen a destacar aquellos tópicos de la filosofía primera que constituyen una solución al problema del no-ser. En particular cabe considerar los siguientes. 1) El establecimiento del principio de no contradicción y su primado por encima del de identidad. 2) La simultánea entronización de la sustancia (como el sentido con relación al cual se dicen los restantes), que es sujeto determinado de predicación, lo que implica la inclusión de la alteridad. 3) El "descubrimiento" de la potencia, que supone inmediatamente la inclusión del no-ser y del tiempo entre lo que es. 4) la definición del movimiento como acto, aunque imperfecto. Al introducir estas aportaciones $-\mathrm{O}$ al realizar es-

1 csegura@filos.ucm.es.

Philosophica, 52, Lisboa, 2018, pp. 141-158. 
tas correcciones- el Estagirita resuelve algunas de las principales aporías en que se encontraba el pensamiento de su tiempo. Al menos en buena medida éstas se derivaban de la indistinción entre atributos y esencias, por lo que fue necesario establecer definitivamente la diferencia entre ser en sí y en otro. Al hacerlo Aristóteles superó los planteamientos monistas y relativistas, dando cabida a la pluralidad, la inidentidad y el tiempo.

Todo lo que se acaba de decir permite aventurar que en la de Aristóteles se puede encontrar el germen de una ontología dinámica. Y ello aunque ésta tenga en su centro la noción de sustancia, pero siempre que esta noción sea correctamente entendida, más allá de las adherencias anquilosantes de la tradición. Lo cierto es que la comprensión fijista del pensamiento aristotélico no hace honor al fundador de la metafísica occidental. En cualquier caso, en este estudio la intención rectora es mostrar que las categorías teóricas elaboradas por Aristóteles sirven, como se ha apuntado al comienzo de estas páginas, para solventar las dificultades relativas al movimiento, la diferencia o el tiempo. Es cierto que el Estagirita proporciona una respuesta a los problemas frente a los que se encuentra que pasa, en definitiva, por arrumbar la que podría ser considerada ya una ontología de la relación. Así es porque el arma definitiva que le permite a Aristóteles dar una respuesta suficiente al problema del no-ser es la articulación de la realidad y el lenguaje en términos de sustancias y accidentes y de sujetos y atributos. De lo que se trata en consecuencia en estas páginas es de hacernos cargo del planteamiento aristotélico teniendo en cuenta, precisamente, que este se establece como alternativa a la entonces imperante ontología de los contrarios.

\section{Consideraciones preliminares}

El preguntar de los primeros pensadores griegos se mantiene vivo y operativo en Aristóteles de modo que su filosofía es, al menos en gran medida, un intento de respuesta, un diálogo con quienes le precedieron. En la senda que conduce hasta el fundador de la metafísica occidental hay un hito que no se puede ignorar. Su nombre es Parménides y la incidencia de su pensamiento continúa siendo imponderable. Al menos en "la senda de la verdad" el pensador de Elea se niega a admitir el cambio y la diferencia y esta negativa pesa decisivamente sobre los que le siguieron, quienes no lograron desembarazarse (aunque lo intentaran) del peso del ser uno. También Sócrates, los sofistas y Platón hubieron de sobrellevar esa pesada carga. Al final de su camino el maestro del Estagirita parece intentar el pa- 
rricidio. Sin embargo, hay razones para creer que, si así fue, no obtuvo un éxito definitivo en su empresa².

El pensamiento de Aristóteles arraiga en ese suelo. Y si bien es cierto que no está dispuesto a renunciar a la consistencia de lo que es, hay que admitir que recoge con energías nuevas y mayor independencia las objeciones que el propio Platón había tomado en consideración y que ya brillaban con tanta fuerza - con el esplendor de lo que se afirma positivamente y no por reacción- en el pensamiento de Heráclito

De manera especialísima se advierte en el discípulo de Platón la insatisfacción que le producen las tentativas de su maestro (esforzadas y valiosas) y la determinación que le lleva a intentar dar una respuesta al inquietante problema, a la oscura cuestión del no-ser ${ }^{3}$. Ése pudo ser el reto de Aristóteles, puesto que lo era el movimiento ${ }^{4}$. Ésa es, al menos, una de

2 Contra la interpretación habitual se pueden encontrar algunos estudios que niegan la tesis del parricidio. Entre otros el de Denis O'Brien, Le non-être. Deux Études sur le Sophiste de Platón, Akademie Verlag, Berlin, 1995, pp. 10-13 y 43-45. A su parecer, "Platon distingue le non-ètre qu'il approuve: la négation exprimant l'altérité, d'avec le non-être qu'il condamme: la négation exprimerait la contrarieté”, p. 17. En el segundo de sus estudios O'Brien desarrolla unas "notes complémentaires", en las que critica los estudios de G. Owen, A. Diés y F.M. Cornford y los de Cornarius y Frede. También en la línea de la negación del parricidio se encuentra la excelente interpretación de Beatriz Bossi, "Back to the Point: Plato and Parmenides - Genuine Parricide?" in Plato's Sophist Revisited, Bossi, B. y Robinson, T.M., (eds.), De Gruyter, Berlin, 2013, pp. 157-174.

3 Tanto M. Narcy como E. Berti han insistido en que la concepción del no-ser en Platón y Aristóteles es muy distinta. Cf. respectivamente: Narcy, M., "La lecture aristotélicienne du Sophiste et ses effets", en Aubenque P. Y Narcy, M. (eds.), Etudes sur le Sophiste du Platon, Bibliopolis, Napoli, 1991 y Berti, Enrico, "Quelques remarques sur le conception aristotélicienne du non-être", en Revue de Philosophie Ancienne, 1 (1983), pp. 115-142. Tal diferencia de concepción ya había sido vista también por Aubenque en su magnífico estudio, Le Problème de l'être chez Aristote: essai sur la problématique aristotélicienne, Presses Universitaires de France, Paris, 1962. Aquí lo citaremos siguiendo la traducción castellana: "Mientras que Platón, a fin de resolver estas últimas dificultades, había opuesto la alteridad al ser, haciendo así de ella un no-ser, Aristóteles, consciente de las contradicciones de la solución platónica y de su incapacidad para dar cuenta del discurso atributivo, restituye la alteridad al ser mismo como uno de sus sentidos (la relación), al tiempo que reconoce semejante alteridad en el lenguaje acerca del ser bajo la forma de una pluralidad de significaciones", en El problema del ser en Aristóteles, Escolar y Mayo, Madrid, 2008.

4 "El movimiento es, para Aristóteles tanto al menos como para Platón, lo que, al separar al ser de sí mismo, introduce en él la negatividad, [pero] también es aquello por medio de lo cual el ser se esfuerza por volver a encontrar su unidad perdida. Fundamento de la escisión, es al mismo tiempo su correctivo", Aubenque, Pierre, El problema del ser en Aristóteles, op. cit., p. 409. 
las formas en que se puede leer el texto de filosofia primera de Aristóteles. Pues bien, sabemos que la solución a ese problema no fue otra que la afirmación de la pluralidad de los sentidos de lo que es. ción y defensa fue posibilitada por la entronización de lo que constituyó su condición de posibilidad: el principio de no contradicción. A la vez, esta comprensión de la multiplicidad va pareja a la prioridad que Aristóteles otorga a la ov̉oía ${ }^{6}$ (posibilitada, justificada y deducida en la mostración del principio de no contradicción). Ahora bien, si Aristóteles entroniza la enti-

5 Metafísica 1060b 32. Es bien conocido el debate de los años sesenta entorno a la polisemia del ser, que arrancó a partir del trabajo de Aubenque recientemente mencionado. Tomás Calvo, ha matizado la interpretación del hermeneuta francés: "La multiplicidad de significaciones del verbo ser no puede considerarse, por tanto, como un caso de mera homonimia o equivocidad. Hay una cierta unidad entre ellas consistente en su referencia común (explícita o implícita) a la entidad o sustancia (ov̉oía)", en Aristóteles y el aristotelismo, Akal, Madrid, 1996, p. 33. El mencionado debate fue continuado por Enrico Berti, en l'unitá del sapere in Aristotele, Cedam, Padova 1965. También la contribución de Patzig a esta cuestión fue determinante al afirmar que no sólo el ser se dice de muchas maneras, sino también la sustancia. Cf., Patzig, Günter, "Theologie und ontologie in der Metaphysik des Aristoteles", Kant-Studien, 52, 1.4 (1961), pp. 185-205. En su paper, "Le statut de l'un et du multiple", publicado en Narcy, Michel y Tordesillas, Alonso (eds.), La "Métaphysique" d'Aristote. Perspectives contemporaines, Vrin, Paris, 2005, Lambros Couloubaritnis sostiene que tal debate alcanzó su punto culminante con W. Leszl, Logic and Metaphysics in Aristotle: Aristotle's treatment of types of equivocity and its relevance to his metaphysical theories, Editrice Antenore, Padova, 1970. Este mismo especialista nos recuerda también el completo estudio de R. Lefebvre sobre esta cuestión. Cf., "L'image onto-théologique de la Métaphysique d'Aristote", Revue de Philosophie Ancienne, 8 (1990), pp. 123-172, cf., p. 19. Pero tampoco se pueden desdeñar los estudios que cito a continuación. Irwin, "Homonymy in Aristotle", Rewiew of Metaphysics, 34 (1981) pp. 523-544, donde su autor interpreta la definición aristotélica de homonimia en el libro de las Categorías de forma amplia, de tal modo que cubre tanto la homonimia accidental como la no accidental. También, Shield, Order in Multiplicity. Homonymy in the Philosophy of Aristotle, Clarendon Press, Oxford, 1999. En esta monografía Shield propone una nueva visión y discusión de las tesis de Aristóteles sobre la homonimia del ser. Por último, de Alejandro Vigo recuerdo dos papers publicados en Estudios Aristotélicos, Eunsa, Pamplona, 2006: "Prioridad y prioridad ontológica según Aristóteles", pp. 23-54 y "Homonimia, explicación y reducción en la Física de Aristóteles”, pp. 213-236.

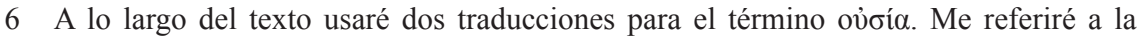
"sustancia" por dos razones: en primer lugar, por lo familiar que nos resulta este término; en segundo lugar y principalmente, porque eso que llamamos "sustancia" ha ocupado como tal sustancia uno de los lugares más destacados en el pensamiento occidental. Pero también utilizaré la traducción de ov̉oía por "entidad," debida a Tomás Calvo Martínez, porque su propuesta me parece no sólo correcta sino, ante todo, acertada para significar con más precisión y libre de adherencias posteriores, lo que Aristóteles quería decir. 
dad no es para reincidir en posibles tópicos parmenídeos sino, al contrario, para dar cabida a los restantes sentidos de lo que es. En definitiva, para dar cabida al no-ser y con él a la pluralidad, la inidentidad, el tiempo y la contingencia, que nos liberan de la prisión de la necesidad ${ }^{7}$.

En este orden de cosas se ha de tener también en cuenta que muchas de las aporías en que se había visto inmerso el pensamiento anterior se derivaban de la indistinción entre atributos y esencias. Basta con recordar que lo último e irreductible a nada anterior era para los primeros filósofos los contrarios y que éstos no eran de nada ni en nada. De acuerdo con ello cabían dos posibilidades: la de los sofistas, que sólo conocían atributos y la de los megáricos, que sólo admitían esencias ${ }^{8}$. Por eso, frente a ambos y para salir de la vía muerta en que se hallaba el pensamiento, era preciso sostener la diferencia entre ser en sí y ser en otro. Pero el establecimiento de esta diferencia -que no sólo hacía inteligible lo real sino que hacía posible el discurso-, implicaba el reconocimiento de la pluralidad.

No cabe duda de que la propuesta aristotélica a la hora de resolver el consagrado problema de lo uno y lo múltiple es novedosa y afortunada: "lo que es se dice de muchas maneras". Lo que es es plural. La unidad es múltiple, aunque articulada. Por eso, sólo articuladamente se accede a

7 Al plantear la pregunta, "Que reste-t-il aujourd'hui de la Métaphysique?, Berti responde en el trabajo que lleva ese título del modo siguiente. "Grâce à son attention pour la multiplicité, la variété, les différences, la mobilité, la métaphysique d'Aristote n'éteint pas la problématicité de l' être, et se révèle en cela d'une extraordinaire actualité (...) Mais néanmoins la question "qu'est-ce-que l'être?", “que signifie être?" ou “qu'est-ce qui explique l'être?" peut encore avoir un sens, si elle n'est pas employée pour mettre de côté la multiplicité, la variété, la problématicité du monde dans lequel nous vivons", en Berti, Enrico, Dialectique, Physique et Métaphysique. Études sur Aristote, Éditions Peeters, Louvain la Neuve, 2008, p. 343.

8 Con relación a este dilema se puede acudir al interesante estudio de Frédérique Ildefonse, "Analyse du langage et analyse de l'être", en Narcy, Michel y Tordesillas, Alonso, La Métaphysique d'Aristote. Perspectives contemporaines, Vrin, Paris, 2005, pp. 183-198. En él este autor concluye que por su teoría de la sustancia, que implica la distinción entre sustancia y accidentes, junto con su teoría de las categorías, que remedia las falsas apariencias de la lexis, Aristóteles contrarresta tanto la disolución heracliteana de las determinaciones cuanto la sustantificación o "calcificación" abusiva, que constituyen las dos expresiones de una paradójica filosofía del accidente; a la vez el Estagirita salvaguarda la identidad. Finalmente, merced a su comprensión de la relación hacia algo uno, detiene el riesgo sofístico sin caer en los abusos de la participación platónica. Cf., p. 198. Vid también en relación con el planteamiento megárico, Charlotte Witt, Ways of Being. Potentiality and Actuality in Aristotle's Metaphysics, Cornell University Press, London, 2003, pp. 17-30. Cf, por último, Aubenque, Pierre, El problema del ser en Aristóteles, op. cit., en particular, p. 140.

9 Metafísica 1003a 33. 
la realidad. Esto supone, llevado hasta el final, que la solución al antiguo problema de la unidad pasa, paradójicamente, por la afirmación de la pluralidad.

Sabemos que la univocidad del ser parmenídeo es de tal naturaleza que impide la pluralidad y, en consecuencia, la predicación, por cuanto el discurso implica pluralidad ${ }^{10}$. Así, la cuestión de lo uno y lo múltiple no es simplemente una cuestión de física u ontológica, sino que es simultáneamente una cuestión lógico-epistemológica: no se plantea aisladamente en el plano de la realidad sino - y con igual radicalidad - en el plano del $\lambda$ óyos, del discurso. Y es preciso articular ambas dimensiones porque, en realidad, ambas resultan inseparables. Se sobreentiende que toda articulación presupone la pluralidad, y que cualquier intento en este sentido ha de dar a aquélla por supuesto. Por eso Parménides no precisa articular y niega que el ser pueda tener predicados, lo que supone la imposibilidad de un $\lambda$ óyos que sea realmente tal.

Los sofistas que defendían la posibilidad del discurso hubieron de hacerlo a costa de postular la existencia de un abismo entre el pensamiento y la realidad: el uno pertenece al ser; la pluralidad al pensar. Por eso, ni al pensamiento ni al lenguaje corresponde nada en la realidad. Algo que Aristóteles ratifica en la Metafísica cuando confirma que: "Platón acertó en cierto modo al decir que la sofística trataba del no-ente. Pues las consideraciones de los sofistas, casi sin excepción, versan sobre el accidente"11, siendo el accidente - como ha dicho inmediatamente antes - un simple nombre ${ }^{12}$. Así, los sofistas se las ven únicamente con nombres y en su ejercicio dialéctico el ser-uno permanece intacto, inalcanzado; no afectado por la división y la pluralidad discursivas que no le pueden alterar.

Éstos, entre otros, son los problemas que plantea el monismo. La solución a ellos se encuentra en la teoría aristotélica de la atribución predicativa. Teoría que a su vez sólo resulta viable desde el gobierno del principio de no contradicción y el correspondiente reconocimiento de la citada pluralidad de los sentidos de lo que es (puesto que presupone inmediatamente la pluralidad de las categorías). Ahora bien, con todas las salvedades, no se puede ignorar que dicha solución estuvo posibilitada por Platón, puesto

10 Con relación al problema del univocismo cf. la crítica de Giovanni Reale en Il concetto di "Filosofia prima" e l'unità della metafísica di Aristotele: con due saggi sui concetti di potenza-atto e di essere, Vita e Pensiero, Milano, 1994. Aquí Reale dedica unas páginas a mostrar la imposibilidad de comprender unívocamente el ser y, así, realiza una crítica al eleatismo, cf., pp. 409-446.

11 Metafísica 1026b 14-15.

12 Cf. Ib., 12-14. 
que fue él quien, de manera especial en el Sofista y el Parménides, planteó por vez primera la cuestión de la atribución; del decir algo como algo. Sin embargo, su teoría de las ideas contenía algunos problemas básicos que no alcanzó a solucionar, concretamente el que se deriva de que, en realidad, las ideas son nombres y, a la vez, sólo hay ideas. A estas dificultades me referiré enseguida, pero antes es preciso que nos detengamos brevemente en lo relativo al principio de no contradicción y a la comprensión no genérica del ser.

\section{La primacía del principio de no contradicción y la ov̉oía}

A mi parecer una de las diferencias fundamentales entre el planteamiento aristotélico y el platónico es que en el segundo todavía se mantiene la primacía del principio de identidad mientras que en el primero el principio que gobierna con carácter principial es el de no contradicción. La razón es que sólo éste permite dar razón de la unidad articulada, esa que sólo es posible a partir del reconocimiento del no-ser. Ahora bien, es evidente que el principio de no contradicción solamente puede comenzar a regir allí donde se ha dado cabida al pensamiento discursivo, pues la unidad no puede ser una pura abstracción mental a partir de la pura dispersión fenoménica, ya que lo que significa el ser de las proposiciones es que algo pertenece a algo. En ellas el ser opera como cópula; su función es atributiva.

Así pues, lo que busca Aristóteles al declarar la vigencia lógico-ontológica del principio de no contradicción es, por una parte, establecer y garantizar la mínima condición de posibilidad del discurso y, por otra parte, como ya se ha dicho, fundamentar la posibilidad de la pluralidad de los sentidos del ser. De hecho, el principio de no contradicción y la pluralidad de sentidos se implican mutuamente. Esto, ya se ha dicho, es lo que evita el monismo y la univocidad.

Aristóteles advierte que es preciso hallar las condiciones mínimas que hagan posible el pensamiento y el lenguaje, porque:

Si las contradicciones son todas simultáneamente verdaderas, dichas de uno mismo, es evidente que todas las cosas serán una sola. Pues será lo mismo una trirreme que un muro o un hombre, si de todo se puede afirmar o negar cualquier cosa, como necesariamente han de admitir los que hacen suyo el razonamiento de Protágoras ${ }^{13}$. 
En efecto, sólo pueden predicarse contrarios de algo que no es nada. Por eso, a juicio de Aristóteles, la ov̉oía es el sujeto de predicación y si no fuera más que un sujeto vacío, no determinado, se podría decir de ella cualquier accidente: uno, varios o todos; pero en este caso la predicación sería accidental. Es decir, los contenidos significativos serían, accidentalmente, accidentes de un conjunto vacío ${ }^{14}$. Aristóteles habla de aquello que define esencialmente y que, por tanto, no es sólo sujeto. En definitiva, si no hubiese sustancia no regiría el principio de no contradicción. La mezcla de Anaxágoras no es más que una pluralidad de contenidos significativos: por eso entiende Aristóteles que, si se niega la sustancia, el lenguaje no es más que manifestación de una multiplicidad pura y el sujeto sólo puede ser admitido por convención. Platón no acabó de ver cómo era posible predicar sin mezclar, pero Aristóteles lo consiguió mediante la distinción entre sujeto y predicado. Para ello entendió que era preciso, además, que dicho sujeto fuera principio.

A partir del principio de no contradicción Aristóteles muestra la necesidad de algo que no sea accidente, pues sin principio ${ }^{15}$ no se podrían predicar los accidentes, ya que éstos son algo de algo. Aristóteles se refiere a la predicación accidental, para sostener que no se pueden decir accidentes de otros accidentes, puesto que todos lo son de una misma sustancia. Esto es: todos se han de decir directamente de ella y no unos de otros. Y es que decir un accidente de otro conduciría a un proceso ad infinitum. Pero tampoco se puede pensar que una sustancia se determina en cuanto tal diciendo de ella sus accidentes, puesto que la sustancia es el sujeto de predicación y tiene que ser ella misma algo determinado. Algo determinado exige sus accidentes, pero no puede depender de ellos, pues si lo hiciera entonces no sería nada de antemano. $\mathrm{Y}$, a su vez, si la sustancia no es nada, entonces la temporalidad ha de ser concebida como puro flujo. Pero Aristóteles también aclara que los accidentes no son lo puramente otro con respecto a la sustancia; que el ser no es tan sólo esta última; también los accidentes son modos de ser. El ser es principio en términos absolutos y este principio integra la diversidad ${ }^{16}$.

14 Para lo relativo a la relación sustancia-predicación cf., Frank A. Lewis, Substance and Predication in Aristotle, Cambridge University Press, Cambridge, 1991. Se trata de un interesante texto en que su autor quiere presentar la Metafísica aristotélica como formando un sistema completo.

15 Estoy refiriendo aquí a la sustancia, a la que se puede y debe, a mi entender, entender también como principio. Así lo hace Jonathan Lear en Aristóteles, Alianza Editorial, Madrid, 1994 (Cambridge, University Press, Cambridge, 1988), p. 279.

16 Tomás Calvo Martínez, entre otros, ha llamado la atención sobre el carácter transcendental que ostenta la argumentación aristotélica acerca del princípio de contradicción y de 


\title{
3. La superación de la consideración genérica del ser
}

He dicho nada más comenzar que la pluralidad de los sentidos del ser resulta nuclear en la Metafísica de Aristóteles y que sólo desde ella se entiende de manera adecuada la precedencia de la sustancia. Cuando Aristóteles se pregunta por lo que es en tanto que algo que es está claro que, en definitiva, se está preguntado por el ser. Desde Parménides se sabía que el ser es tò hén: lo uno. Y sin embargo, Aristóteles dice precisamente que el ser es muchos y que es plural. Añade, además, que Parménides se equivocó en cuanto a la esencia del ser al pensar que era absolutamente uno:

\begin{abstract}
Pero evidentemente no es verdad que, si 'ser' sólo significa una cosa y no es posible al mismo tiempo la contradicción, entonces el no-ser no es. Porque nada impide que haya, no el no-ser absoluto, sino un cierto no-ser. Por otra parte, es absurdo decir que Todo es uno porque no puede haber nada fuera del ser mismo [auto tò ón]. Pues ¿qué se ha de entender por el ser mismo sino 'lo que propiamente es'? Pero si esto es así, nada impide que las cosas sean múltiples. Es evidente, entonces, que el ser no puede ser uno en este sentido ${ }^{17}$.
\end{abstract}

Como prueba en contra de este monismo Aristóteles acude a las categorías. Lo cierto es que la pluralidad no desplaza la unidad y que con el Estagirita la cuestión de lo que es como uno gana toda su profundidad. Ya Platón había dado un paso en este sentido al afirmar que lo no-ente había de ser también introducido en el ámbito de lo que es. Así es como el maestro de Aristóteles se atrevía valerosamente a dar entrada a la negatividad y por lo tanto a la pluralidad: en primer lugar, porque lo uno ya no está separado

la sustancia. Así en "Démostration de l'ousia et foundation des sciences", en Narcy, Michel y Tordesillas, Alonso (eds.), La "Métaphysique" d'Aristote. Perspectives contemporaines, op. cit., pp. 171-182. Para lo relativo a la deducción de la sustancia como sujeto y a la problemática de la predicación se puede consultar también el estudio de Claudia Carbonell, Movimiento y forma en Aristóteles, Eunsa, Pamplona, 2007, pp. 36-46. Por su parte Jonathan Lear ha insistido en el empeño aristotélico por mostrar que la estructura de la realidad determina la de nuestro pensamiento y que ésta es la razón por la que el Estagirita recurre a la sustancia. Así, "el hecho mismo de que el mundo se componga de sustancias y propiedades nos obliga a pensar, a hablar, y a actuar de determinadas formas. En un mundo compuesto de sustancias, un pensador tiene que ser alguien que crea en el principio de no-contradicción (...). Es porque la sustancia es la base de la realidad, por lo que los pensadores somos capaces de realizar una investigación general sobre la sustancias. Es decir, somos capaces de ser filósofos comprometidos en una investigación metafísica”. Lear, J., Aristóteles, op. cit. Cf. por último, Terence Irwin, Aristotle's First Principles, Clarendon Press, Oxford, 1988; cf. en particular la parte dedicada a "The science of Being", pp. 79-198.

17 Física 187a 1-10. 
de lo múltiple. En segundo lugar porque se declara la copertenencia de ambos. En tercer lugar porque el ser como uno es en sí múltiple, de manera que lo que es se dice en varios sentidos, pero no de un único modo.

Acabo de recordar que esto no supone la ruptura de la unidad del uno, y es que para cada ente hay una referencia a un cierto uno y común: "pues si se dice algo común cae bajo una misma ciencia"18. Sabemos que Aristóteles aclara desde el primer momento que el ser va a ser dicho con referencia a algo común, de tal forma que lo que es no pierde la unidad por la multiplicidad. Uno y ser son dos conceptos diferentes, pero según la esencia son lo mismo ${ }^{19}$. A la vez, a la esencia del ser pertenece absolutamente lo uno y en la unidad está ya siempre el ser. Por ello sostiene también Aristóteles que el ser y la unidad se dicen, igualmente, de muchas maneras ${ }^{20}$. Ahora bien, la unidad de la que habla Aristóteles, como el ser, no es genérica. El ser no es dicho como sinónimo, y el mismo Aristóteles demuestra qué es lo que pasaría si lo fuera. En primer lugar, el género no puede contener sus diferencias. En segundo lugar, cada tipo perteneciente a un género añade algo que el género no tiene. Por último, el ser no es un género, por lo tanto tampoco un concepto y, en consecuencia, no es posible la definición.

Pero ya sabemos que existe un tipo de relación distinta de la del género: la salud se dice de muchas maneras distintas, pero los sentidos secundarios de la salud co-mencionan, co-significan, el sentido principal ${ }^{21}$. De igual modo podemos decir de la unidad que existe entre los diversos sentidos del $\operatorname{ser}^{22}$. Hay un primer significado con relación al cual se dicen los restantes; así, los otros corresponden al primer significado bajo distintos aspectos. Se trata del pros hén al que tantas veces apeló el Estagirita y después sus discípulos a lo largo de la historia. Por tanto, no hay un significado general que planee sobre los demás; hay un primero, un uno a través del cual todos los demás pueden ser dichos ${ }^{23}$ : éste es el significado fundamental y directo. Ésta es la $\grave{\alpha} \rho \chi \eta ́$, el principio. Por eso precisa Aristóteles que: "todo lo que es se dice con relación a un solo principio"24. La ả $\rho \eta \dot{~ e s ~}$ lo unificante de lo plural que le pertenece. Se trata por lo tanto de un $\lambda \dot{\varepsilon} \gamma \varepsilon ı v$

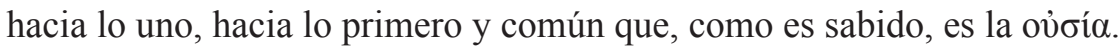

Se ha subrayado siempre que la concepción aristotélica del ser, fren-

18 Metafísica 1061a 10ss y también Ib., 1060b 35: "Pues si se dice según algo común cae bajo una sola ciencia".

19 Cf. Ib., 1003b 22 -1004a2; Ib., 1061a 15-18.

20 Cf. Ib., 1053b 25.

21 Cf. Ib., 1003a 33-16.

22 Cf. Ib., 1018a 12-15.

23 Cf. $I b ., 1004$ a 25.

$24 I b ., 1003 \mathrm{~b} 6$. 
te a la platónica, no es genérica. Algo que Aristóteles supera limpiamente, pudiendo solucionar los problemas que se le plantearon a su maestro - principalmente respecto de la unidad en el mundo de las ideas- por concebirla en tales términos. En efecto, Platón no acaba de solucionar el problema de la identidad y la diferencia, puesto que el género lo es en la medida en que contiene lo diverso. Por otra parte, si el ser es género entonces no caben sentidos tales como la potencia y el acto o el ser coincidental. De las dificultades platónicas pasaré a ocuparme en el siguiente apartado. Antes importa subrayar que si Aristóteles se aferra a la referencia a algo uno es porque ésta admite la pluralidad sin romper la unidad y evita tanto

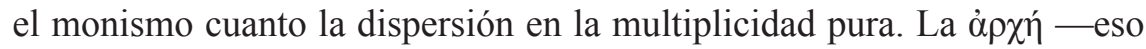
uno con relación al cual se dicen los demás- es el significado directivo que funda los demás.

\section{La disputa de Aristóteles con Platón: lo en sí y lo otro}

Es indudable que uno de los grandes hallazgos de Platón es el del concepto y la definición: cada idea ha de tener un determinado y exclusivo contenido semántico; ha de significar, de manera definida y concreta, lo que significa y nada distinto. Pero sabemos que este planteamiento está plagado de dificultades, pues ¿cuál es el estatuto ontológico de la idea? ¿Es la idea algo subsistente en sí o es más bien algo semejante a un atributo? Si las ideas son lo real y eterno no sólo se ha de plantear, como hace Platón en el Sofista, el modo en que unas puedan comunicar con otras, sino también la pregunta, ¿acerca de qué se dicen dichas ideas?, ¿o es que se dicen entre sí? Inmediatamente se advierte que este planteamiento conduciría a la conclusión de que todas las ideas no son sino accidentes, en cuyo caso ni la ciencia dialéctica, con todo su rigor, podría vencer al sofista. Pero, además, ¿es posible que las ideas se prediquen de lo contingente? Es evidente que carece de sentido que las esencias, lo realmente verdadero, se diga de lo contingente y mudable ${ }^{25}$.

Sea como fuere, se mantiene el problema relativo al sujeto del decir.

25 Para la problemática propia del Sofista se pueden ver entre otros la colección de ensayos publicada por Aubenque, P. y Narcy, M. (eds.), Études sur le Sophiste de Platón, Napoles, Bibliopolis, 1991, el ya citado de Denis O'Brien, Le non-être. Deux éstudes sur le Sophiste de Platón y la colección de ensayos editados por Beatriz Bossi y T.M. Robinson, también citada: Plato's Sophist Revisited. Además, de Paul Seligman, Being and not-being. An Introduction to Plato's Sophist, Martinus Nijhoff, The Hague, 2012. 
Y es que mientras que no se advierta que el sujeto es entidad, la cuestión queda sin resolver. Así, por ejemplo, la idea de "identidad" ¿es? Porque parece que ha de ser, pero si es, entonces no es simplemente la identidad, puesto que además participa de la idea de ser, y entonces no es idéntica a sí misma de manera perfecta. En el Sofista Platón muestra sin reparos las dificultades en que se encuentra su pensamiento y busca con ahínco la comunicación entre los géneros supremos creyendo encontrar en ella una solución. Pero ya sabemos -y lo acabo de apuntar con un mero ejemplo-que tampoco la comunicación entre las ideas resolvía enteramente las dificultades ${ }^{26}$.

El problema estriba, precisamente, en que las ideas son géneros. Pero lo son como los nueve géneros supremos establecidos por Aristóteles que no son en sí sino en otro. Es decir, como accidentes. Aunque el Sofista es un diálogo extraordinario en el que Platón hace avanzar decidida y brillantemente la teoría de la predicación atributiva, del discurso apofántico, sin embargo, la carencia de algo así como la ov̉oía -la ausencia de un sujeto determinado-impide que el maestro de Sócrates solvente adecuadamente todos los problemas. Aristóteles entiende que si no se puede atribuir a algo que exija unas determinaciones y no otras, que si no hay algo que sea no sólo sujeto indiferenciado de predicación sino determinado, ov̉oía, entonces lo único que se puede decir es lo que dijeron los sofistas: que el pensamiento nada tiene que ver con la realidad; que la predicación es

A estos estudios hay que añadir dos monografías de F. Mié, pues como ha señalado E. H. Monbello, las dos obras de Mié que se citan a continuación merecen ser consideradas como una herramienta de consulta para los intérpretes de la filosofía de Platón. Se trata de: Mié, F., Dialéctica, predicación y metafisica en Platón. Investigaciones sobre el Sofista y los diálogos tardios, Ediciones del Copista, Córdoba, 2004 y Lenguaje, conocimiento y realidad en la teoría de las ideas de Platón: investigaciones sobre los diálogos medios, Ediciones del Copista, Córdoba, 2004. En el primero de los textos citados, Mié da cuenta de las interpretaciones más usuales y conocidas de la semántica del verbo ser así como de las implicaciones que ésta tiene para la interpretación de la doctrina platónica sobre el no ser -como las de Crombie, Cornford, Runcinam y Moravcsik- presentada en el Sofista. Cf., Monbello, "of Fabián Mié, Dialéctica, predicación y metafísica en Platón: investigaciones sobre el Sofista y los diálogos tardios", en Plato. Le Journal Internet de la Société Platonicienne Internationale, 6 (2006). Cf. también Segura, Carmen, "La respuesta al Sofista de Platón en la Metafísica IV de Aristóteles”, en Padrón, Héctor Jorge (ed.), Aristóteles, Facultad de Filosofía y Letras de la Universidad Nacional de Cuyo, Mendoza, 1997, pp. 86-96.

26 Con relación a la comunicación entre las ideas cf., Fronterrota, F., "La notion de

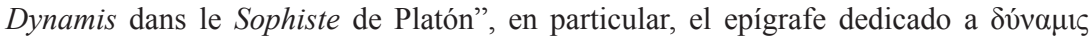
y Korvovía (pp, 201-207), en Michel Crubellier, et alia (eds.), Dynamis. Autour de la puissance chez Aristote, Louvain La Neuve, éditións Peeters, 2008, pp. 187-224. 
accidental.

Acabo de decir que las ideas platónicas operaran como "lo que se predica de...", es decir, como simples atributos. Pues bien, esta dificultad se agrava hasta el extremo si, como sostiene Aristóteles, cabe que cada idea, o forma, no se identifique con su esencia ${ }^{27}$.

Y de los que afirman la existencia de las ideas, unos dicen que la Díada se identifica con la Línea en sí y otros que con la Forma de la Línea, ya que en ciertos casos la Forma y aquello de que es la Forma son lo mismo (así, la Díada y la Forma de la Díada), pero no es así en el caso de la Línea. Con que sucede que hay una única Forma de una pluralidad de cosas cuya forma es manifiestamente distinta (esto les sucedió también a los Pitagóricos), y resulta posible establecer que la Forma de todas las cosas es una y la misma, y que las demás no son Formas. Desde luego, así todas las cosas serían una sola ${ }^{28}$.

Pero entonces no es sólo que todas las cosas sean una, sino que ya no se puede afirmar que las ideas sean lo en sí. Ahora bien, está claro que en un universo donde impera el modelo técnico, donde lo sensible no es más que copia de lo inteligible, dichos arquetipos deberían ser en sí; de lo contrario no serían arquetipos; no pasarían de ser sino un espejismo, algo más irreal que el mismo mundo sensible. La multiplicidad, entendida como pura dispersión sin inteligibilidad posible, se daría ya en el mundo de las ideas que por tanto sería, él mismo, ininteligible.

Para eludir estas contradicciones se podría plantear otra hipótesis, que al final resulta más absurda, aunque de alguna manera se deja perfilar a partir de la observación aristotélica según la cual "la forma de todas las cosas es una y la misma". Se trataría de afirmar, en efecto, la posibilidad de un sujeto único, anterior, una especie de "supra idea" que fuera ella sólo el fundamento de la determinación, pero entonces, de nuevo, las demás no serían más que sus diversas y plurales manifestaciones. Pero no parece que fuera esto lo que se proponía Platón, quien a la vez quería defender la unidad del ser uno, con Parménides, y dar razón de la pluralidad (tarea que aborda particularmente en el Sofista). Para defender lo primero es para lo que sostiene que cada idea es en sí. Pero entonces vuelven a plantearse los problemas ya enunciados.

Aristóteles advierte que no se puede pretender una pluralidad de géneros supremos absolutamente separados y con idéntica jerarquía ontológica; que no todos pueden ser accidentes. El Estagirita admite de buen grado que puede haber accidentes, pero entiende que ha de haber también 
oủoía: un fundamento que los accidentes no son, porque no son en sí. Si lo fueran entonces no habría sustancia, pero si la hay, entonces los accidentes son de ella y no indiscriminadamente sino como atributos, como predicados. Han de ser atribuidos y además por algo que no sea uno de ellos ni de su mismo orden, por muy trascendental que quiera ser. De lo contrario se plantea el problema que Platón advierte en el Parménides: si el uno es el uno, entonces no es el $\mathrm{ser}^{29}$. Por eso el Estagirita descubre que es preciso unir las ideas, pero de una forma que no sea arbitraria. Precisamente esto es lo que garantiza la introducción aristotélica del principio de no contradicción que - teniendo en cuenta que los accidentes no son absolutamente lo otro que; el no-ente - une proposicionalmente, estableciendo pertenencias que resulten suficientes.

Es cierto que Platón quiere atender a la pluralidad y que para hacerlo toma en consideración, como no podría ser de otro modo, a lo otro, al no-ser. Lo otro es el no de aquello a lo que hay que referirlo: al ser. $L o$ otro es así, lo otro de lo mismo, hallándose por tanto su determinación, su mismidad, fuera ${ }^{30}$. Sin embargo, y precisamente por ello, lo otro no puede desligarse de lo mismo; esto sería un puro desperdigamiento, supondría la mera desintegración. En síntesis: se puede advertir que en la formulación platónica del Sofista relativa a lo otro hay ya un conato de atribución, en cuanto que en ésta el accidente es lo otro del sujeto: es en otro, es lo otro del ente, que no es independiente, porque sólo así puede ser. Pero el juicio,

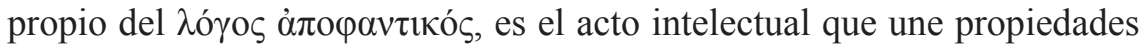
a entidades $-\mathrm{a}$ algo que es un $\tau o ́ \delta \varepsilon \tau-\mathrm{y}$, estrictamente, como ya hemos dicho, Platón no podía hacerlo porque no tenía un sujeto que operase como tal. No tenía un sujeto-entidad primera al que referir los contenidos significativos de las ideas.

Lo que ocurre, en definitiva, es que si la concepción del ser es gené-

29 La solución aristotélica a esta cuestión, por ejemplo, en Metafísica 1003b 20.

30 Fronterrota subraya en el artículo ya citado que en el Sofista de Platón se puede hallar una ontología de la comunicación de los géneros y de una concepción dinámica y participativa del ser, lo que sólo es posible en virtud de la idea de diferencia. Por otra parte, Fonterrota señala especialmente que se puede constatar que la noción de $\delta v ́ v \alpha \mu \iota$, , situada en la base de la concepción ontológica del Sofista, implica ya, por ella misma, una forma de alteridad. Esto le permite hablar a Fonterrota de una "ontología dinámica" en el Sofista, que se encontraría fundada en la categoría de la diferencia, puesto que es su condición necesaria. Cf., "La notion de Dynamis dans le Sophiste de Platón”, op. cit., pp. 210-211. Cabe pensar que tal "ontología dinámica" podría estar próxima a las actuales “ontologías de la relación”. Vid. también del mismo autor, "L' être et la participation de l'autre. Une nouvelle ontologie dans le Sophiste", Les Études philosophiques, 3 (1995), pp. 311-353 y Platone, Sofista, RCS Rizzoli BUR, Milano, 2007. 
rica, entonces no cabe la predicación. Por eso, Aristóteles da un paso más. Éste viene posibilitado por su concepción misma de lo que es, y por la solución que él da al problema del fundamento, en el sentido de que éste no es único, ni se encuentra separado de aquello que funda. Por el contrario: cada ser con capacidad de automovimiento es $\varphi v ́ \sigma ı \varsigma$, naturaleza, principio para sí mismo. Aristóteles multiplica así las ả $\rho \chi \alpha$, anulando con ello cualquier planteamiento monista de la realidad y acabando simultáneamente (aunque no fuera así a juicio de Heidegger) con la vigencia del modelo técnico de Platón. La púఠıৎ es ahora sustancia, o mejor dicho sustancias. La filosofía primera, la ciencia que se busca es de lo que es en tanto que es, y esto significa para Aristóteles, غ̇ंı

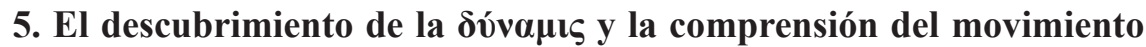

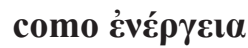

Se podría entender, después de lo que se acaba de decir, que el planteamiento aristotélico es insuficiente a la hora de resolver los problemas relativos al movimiento, el tiempo y la diferencia, puesto que en la centralidad de la sustancia se podría ver un cierto inmovilismo o fijismo, como si la de Aristóteles fuese una "ontología estática". Sin embargo no se puede olvidar que es el autor de la Metafísica el que descubre y da cabida a la potencia -que no sólo ha de ser entendida como pasividad sino también

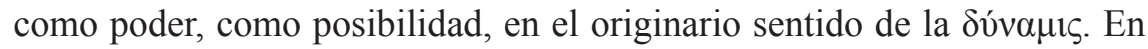
mi opinión, la toma en consideración de la potencia supone la posibilidad de ampliación del criterio presencial e implica, por tanto, un modo "flexible" de interpretar la entidad y nuestro modo de decirla.

Así es, incluyendo la potencia Aristóteles está, por lo mismo, incluyendo el tiempo, pero no al modo en que lo hizo Heráclito. Para Aristóteles la unificación es posible por la potencia, que ahora es real, aunque no esté presente (es decir, la posibilidad es también actual, por tanto fundada, aunque no se trate de una actualidad perfecta); lo cual significaría que la presencia -el pleno estar ahí delante- ya no es, para el Estagirita, el criterio definitivo y único. También quizá Heráclito lo intuía de algún modo y por

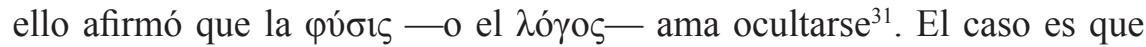
Aristóteles entiende que hay que distinguir la presencia y el fundamento, lo que le permite dar carta de naturaleza al tiempo y la potencia. Es decir,

31 Heráclito, fr. 123. 
al no-ser.

No cabe duda que para Aristóteles lo auténtica y perfectamente real es aquello que es acto o está en acto, que la potencia sólo es en la medida en que se ordena al acto ${ }^{32}$. Pero tampoco conviene olvidar que para el Estagirita el movimiento es acto: "la actualidad de lo potencial en tanto que tal" 33 . Es decir, que a juicio de Aristóteles -aunque él mismo confiesa que es difícil de ver, aunque posible que sea- hasta lo potencial en tanto que tal tiene su propia y peculiar actualidad. El mismo Estagirita manifiesta con relación al movimiento que,

Algunos lo definen como diversidad, como desigualdad y como 'algo que no es': ahora bien, a nada de esto le corresponde necesariamente moverse, pero es que tampoco el cambio tiene lugar ni hacia estas cosas ni a partir de ellas con más razón que a partir de sus opuestos. Y la razón de que lo reduzcan a estas cosas está en que el movimiento parece ser algo indeterminado (...) Y la causa de que el movimiento aparezca como indeterminado es que no resulta posible reducirlo ni a la potencia ni al acto de las cosas que son"34.

Por esta razón, como también concluye el mismo Aristóteles, "el movimiento parece ser cierto tipo de actualización, pero incompleta"35. El movimiento no es, por tanto, acto perfecto, pero es. De este modo resuelve ahora Aristóteles el problema del no-ser. Por una parte, la explicación aristotélica del movimiento se fundamenta en y despliega la diferencia entre no-ser actual y no-ser potencial. Una diferencia que se apoya en la primera básica y principal, ya destacada por Platón: la que existe entre no-ser absoluto (al que el maestro de Aristóteles declara en el Sofista haberle dado la espalda hace ya mucho) y el no-ser relativo. Por otra parte, el Estagirita

32 Cf., Jonahthan B. Beere, "The priority in Being of Energeia", en Michel Crubellier et alia (eds.), Dynamis. Autour de la puissance chez Aristote, op. cit., pp. 429-456. En este trabajo, recordando la doctrina aristotélica según la cual el acto es anterior a la potencia en cuanto a la noción, en cuanto a la entidad y en cuanto al tiempo (Cf., Metafísica IX, 1049b3 - 1051a3), Beere concluirá que, si aceptamos la distinción aristotélica entre ser en potencia y ser en acto, "Then he has given us powerful arguments to agree that the ultimate principles of being must be energeiai that are not the exercise of any capacity", p. 455. Respecto a esta misma cuestión cf. también Charlotte Witt, Ways of Being. Potentiality and Actuality in Aristotle's Metaphysics, Cornell University Press, London, 2003, pp. 75-96.

33 Física 201a 10-11; Ib., 27-28 e Ib., 201b5. Para lo relativo al movimiento y especialmente a la peculiaridad de la relación entre acto y potencia, cf., Carbonell, Claudia, Movimiento y forma en Aristóteles, op. cit., pp. 95-138.

34 Metafisica 1066a 11-19.

$35 \mathrm{Ib} ., 21-22$. 
explica que el movimiento no es no-ser, que por el contrario es y que, por tanto, cabe ocuparse y dar cuenta de él ${ }^{36}$.

Pues bien, precisamente en la declaración según la cual el movi-

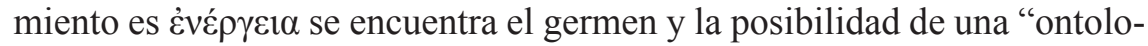
gía dinámica". No en balde Aristóteles observa que: "la palabra 'acto', vinculada a la realización plena ( $\dot{\varepsilon} v \tau \varepsilon \lambda \dot{\varepsilon} \chi \varepsilon 1 \alpha)$, se ha extendido también a otras cosas, fundamentalmente a partir de los movimientos. En efecto, parece

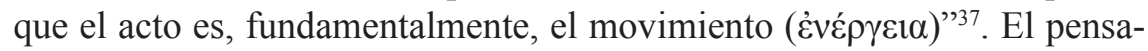
miento anterior a Aristóteles había encontrado en el movimiento la piedra de escándalo para la filosofía y lo había reducido a no-ser. Al declarar que el acto es fundamentalmente el movimiento, nuestro pensador aleja a este último del oscuro mundo de lo indecible e incomunicable.

\section{RESUMEN}

36 A este respecto Cf, el artículo de S. Borade, "Où se trouve l'activité?, En Dynamis. Autour de la puissance chez Aristote, op. cit., pp. 457-471. Este estudio aparece también en Being, Nature, and Life in Aristotle, Cambridge University Press, Cambridge, 2010, pp. 198-212. En este trabajo la autora destaca el carácter de acto del movimiento al glosar el ejemplo aristotélico de la construcción de la casa (Metafísica, 1050a 25-6a 30-35). Así, sostiene que la actividad transitiva posee un status télico superior al de la dynamis correspondiente, cf., p. 469. En concreto afirma lo que sigue. "L'energeia en cours de la construction a lieu pour la maison, mais elle a lieu pareillement pour que son mode ayant été réalisé s'ensuive. D'une certaine façon, alors, cette energeia a lieu pour elle-même", p. 469. También se ocupa del movimiento y su particular estatuto ontológico M. L. Gill en Aristotle on Substance, Princeton University Press, Princenton, 1989. En esta obra consagrada al problema de la unidad en las sustancias materiales, Gill se ocupa de la causa del devenir, del movimiento y su definición, cf., pp. 172211 (más específicamente pp. 183-194). Para la definición aristotélica de movimiento también interesa L. A. Kosman, “Aristotle's Definition of Motion” en Phronesis, 14, 1 (1969), pp. 40-62.

37 Metafísica 1047a 30-32. 
El objetivo de esta investigación es proponer una lectura de la Metafísica de Aristóteles entendida como respuesta al problema del no-ser. Esta orientación revelará la vigencia de los planteamientos ontológicos aristotélicos para el presente, porque también hoy el movimiento, la diferencia, la inidentidad y el tiempo constituyen problemas filosóficos fundamentales. Sabemos que Aristóteles elaboró su ontología en diálogo y discusión con quienes le precedieron. En este trabajo apunto a determinados aspectos de ese debate en la medida que contribuyen a destacar aquellos tópicos de la filosofía primera que constituyen una solución al problema del no-ser.

Palabras clave: Aristóteles - Metafísica - no-ser - principio de no contradicción - Sustancia - potencia.

\begin{abstract}
The aim of this research is to propose a reading of Aristotle's Metaphysics understood as an answer to the problem of non-being. This orientation will reveal the validity of the Aristotelian ontological approaches for the present, because today also the movement, the difference, the inidentity and the time constitute fundamental philosophical problems. We know that Aristotle displayed his ontology in dialogue and discussion with his predecessors. In this paper, I point out certain aspects of this debate to the extent that they contribute to highlighting those topics of the first philosophy that constitute a solution to the problem of non-being.
\end{abstract}

Keywords: Aristoteles - metaphysics - non-being - principle of non-contradiction - Substance - potentiality. 
EDITORIAL

ARTIGOS

AT THE HEART OF A DECISION IS A NARRATIVE

R. M. Zaner

AGONIA E RAZÕES PARA AGIR: UMA CRÍTICA A PARFIT

Pedro Galvão

A PROPÓSITO DA NATURALIZAÇÃO DA DOR NA OBRA DE FILIPE MONTALTO

Manuel Silvério Marques e José Morgado Pereira

AS MEDITAÇÕES SOBRE AS LÁGRIMAS E O CHORO DE JOHAN FRIEDRICH SCHREIBER

Palmira Fontes da Costa

REDESCOBRIR A SAÚDE QUE NUNCA SE PERDEU. DOENÇA, SOFRIMENTO E CURA NO BUDISMO

Paulo Borges

COMPREENDER A DOR. A PROPÓSITO DE UM CASO DE ANOREXIA NERVOSA CRÓNICA

Dulce Bouça

O PASSO DO ABISMO: O DESVIVER, A AGONIA E A MORTE DIGNA

Manuel Silvério Marques

A “BOA MORTE” DE BACON

António Lourenço Marques

SEDAÇÃO PALIATIVA, PERSPETIVA DE UM CLÍNICO

Madalena Feio

ENSAIOS

LA RESPUESTA AL PROBLEMA DEL NO-SER EN LA METAFÍSICA DE ARISTÓTELES

Maria Carmen Segura Peraita

GRAMSCI E I QUADERNI: FILOSOFIA DELLA PRASSI E IMMANENZA TRA MATERIALISMO E IDEALISMO

Luca Onesti

PRÉMIO PROF. DOUTOR JOAQUIM CERQUEIRA GONÇALVES PARA ALUNOS

DO 1. ${ }^{\circ}$ CICLO/ CURSOS DE LICENCIATURA (Edição de 2018)

KANT NO JARDIM DO NÃO SEI QUÊ. NOTAS AO PARÁGRAFO §49

DA CRÍTICA DA FACULDADE DO JUÍZO

João Maria Carvalho

DISSERTAÇÕES

LUDO-ESTÉTICA

Pedro Miguel Celestino Pereira

RECENSÕES

THOMAS P. KASULIS, ENGAGING JAPANESE PHILOSOPHY:

A SHORT HISTORY, HONOLULU, UNIVERSITY OF HAWAI'I PRESS, 2018

Ricardo Santos Alexandre

INSTRUÇÕES AOS AUTORES - NORMAS DE PUBLICAÇÃO

INSTRUCTIONS TO AUTHORS - PUBLICATION PROCEDURES

*

\section{Patrocínios}

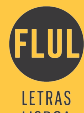

LISBOA

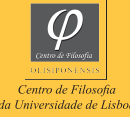

FCT 